\title{
Archéopages
}

Archéopages

Archéologie et société

\section{La mode de l'animal exotique dans la haute société gallo-romaine. Sépulture d'un singe dans la nécropole de la rue des Caillons à Poitiers}

Frédéric Gerber et Anna Baudry-Dautry

\section{(2) OpenEdition}

Journals

Édition électronique

URL : https://journals.openedition.org/archeopages/296

DOI : 10.4000/archeopages.296

ISSN : 2269-9872

Éditeur

INRAP - Institut national de recherches archéologiques préventives

Édition imprimée

Date de publication : 1 mai 2013

Pagination : $42-47$

ISSN : $1622-8545$

\section{Référence électronique}

Frédéric Gerber et Anna Baudry-Dautry, «La mode de l'animal exotique dans la haute société galloromaine. Sépulture d'un singe dans la nécropole de la rue des Caillons à Poitiers », Archéopages [En ligne], 35| 10/2012, mis en ligne le 01 octobre 2014, consulté le 21 janvier 2022. URL : http:// journals.openedition.org/archeopages/296 ; DOI : https://doi.org/10.4000/archeopages.296 


\title{
La mode de l'animal exotique \\ dans la haute société gallo-romaine Sépulture d'un singe dans la nécropole de la rue des Caillons à Poitiers
}

\author{
Frédéric Gerber Inrap \\ Anna Baudry-Dautry Inrap
}

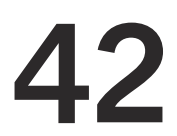

1. La fouille venant tout juste de se terminer, il reste à confirmer que les esquilles d'os brûlés sont bien humaines et à affiner les datations. 2. De nombreuses informations sur les rituels funéraires, et notamment les banquets, ont été collectées et sont en cours d'étude, apportant déjà un éclairage nouveau sur le sujet.

3.Créées par le zoologiste britannique John Edward Gray en 1821.

\section{La nécropole}

Jusqu'au milieu du $\mathrm{III}^{\mathrm{e}} \mathrm{s}$., la zone située entre la ville antique de Lemonum, aujourd'hui Poitiers (Vienne), et le Clain semble être restée non bâtie. L'occupation de l'actuel terrain, délimité par la rue des Caillons, est caractérisée par quelques fosses témoignant d'une utilisation probablement agricole des lieux, alors que des chablis indiquent la présence ancienne d'arbres. Survient ensuite un apport conséquent de remblais correspondant à l'aménagement de terrasses entre la rupture de pente (qui marque la limite urbaine) et la rivière. Un chemin empierré terminé par une sorte d'esplanade conduit à un ensemble funéraire qui est implanté alors en rive gauche du Clain. Un petit édicule quadrangulaire d'environ $1 \mathrm{~m}^{2}$ semble marquer la première sépulture. Il abrite les cendres du défunt, répandues dans la niche centrale de la construction scellée par une simple dalle de terre cuite. C'est le seul témoignage du rituel de l'incinération sur ce site. Son niveau de démolition a livré la base d'une statue, probable héritage de famille, représentant les jambes d'un personnage assis en tailleur sur un coussin, rappelant très fortement les sculptures d'Argentomagus, l'actuelle ville d'Argenton-surCreuse (Indre).

Cinquante trois tombes ont ensuite été implantées à proximité, durant la seconde moitié du $\mathrm{III}^{\mathrm{e}} \mathrm{s}$. Elles pourraient appartenir à un même groupe «familial». Les deux sexes et toutes les tranches d'âges sont représentés. Mis à part quelques rares vases et gobelets en verre, ainsi que des monnaies déposées soit sur les yeux du mort soit dans une de ses mains, le mobilier funéraire est essentiellement composé de bijoux (colliers en perles, bracelets en perles, bronze et lignite, fibules en bronze, épingles en os) ${ }^{2}$. Un enclos funéraire quadrangulaire de $63 \mathrm{~m}^{2}$ a été installé au même endroit, tardivement (fin du III ${ }^{\mathrm{e}}$-début du IV ${ }^{\mathrm{e}} \mathrm{s}$.). Il recoupe plusieurs sépultures. Fortement arasé et recoupé par plusieurs aménagements médiévaux, ni son élévation ni son accès ne sont connus [ill. 1]. Une grande fosse quadrangulaire abritait probablement le sarcophage de son propriétaire, comme c'est le cas pour des enclos fouillés récemment dans la région. Si ce dernier a été récupéré, la tombe voisine, quant à elle, a été préservée. Découverte exceptionnelle, il s'agit de la sépulture d'un petit singe. Elle témoigne de l'importance donnée ici à cet animal exotique, prisé comme animal de compagnie dans les familles aisées romaines et gallo-romaines.

\section{La tombe du singe}

Elle se présente sous la forme d'une simple fosse ovale de 0,90 sur $1,15 \mathrm{~m}$, et profonde de 0,20 à $0,30 \mathrm{~m}$. Les parois sont verticales et le fond plat. Le squelette de l'animal est couché sur le flanc droit dans le sens de la largeur de la tombe, suivant une orientation sud-ouest/nord-est [ill. 2]. Il est entouré de blocs calcaires et de fragments de briques et recouvert d'un grand nombre de blocs et de moellons calcaires laissant supposer la présence d'un empierrement sur un cercueil de bois. On retrouve le même aménagement dans plusieurs tombes de nourrissons fouillées sur le site dans les niveaux antérieurs à l'enclos funéraire. La sépulture était recouverte par les éléments de démolition de cet enclos. Létude du squelette est en cours, mais les premières observations macroscopiques des ossements le rattachent à la famille des Cercopithecidae et à la sous-famille des Cercopithecinae $\mathbf{3}^{\mathbf{3}}$. Les stades d'épiphysation des ossements associés aux éruptions dentaires attestent qu'il s'agitd'un individu adulte. 
1. Vue d'ensemble de l'enclos funéraire de la rue des Caillons

à Poitiers. On distingue

la tombe du petit singe

disposée juste à côté

d'une grande fosse qui aurait

pu abriter le sarcophage

du propriétaire de l'enclos.

2. Sépulture de singe

découverte dans les niveaux

d'utilisation de l'enclos

funéraire.

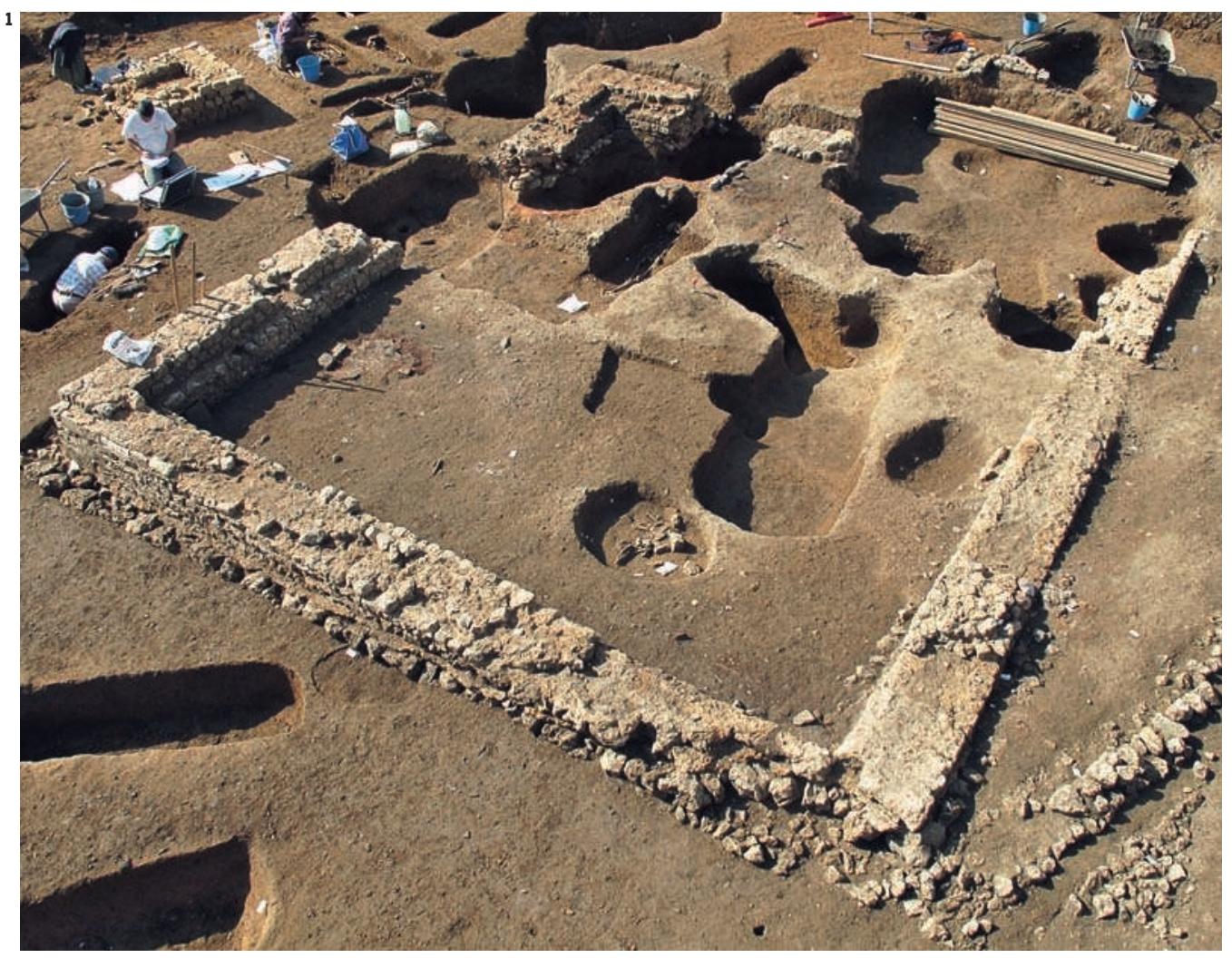

ஜ

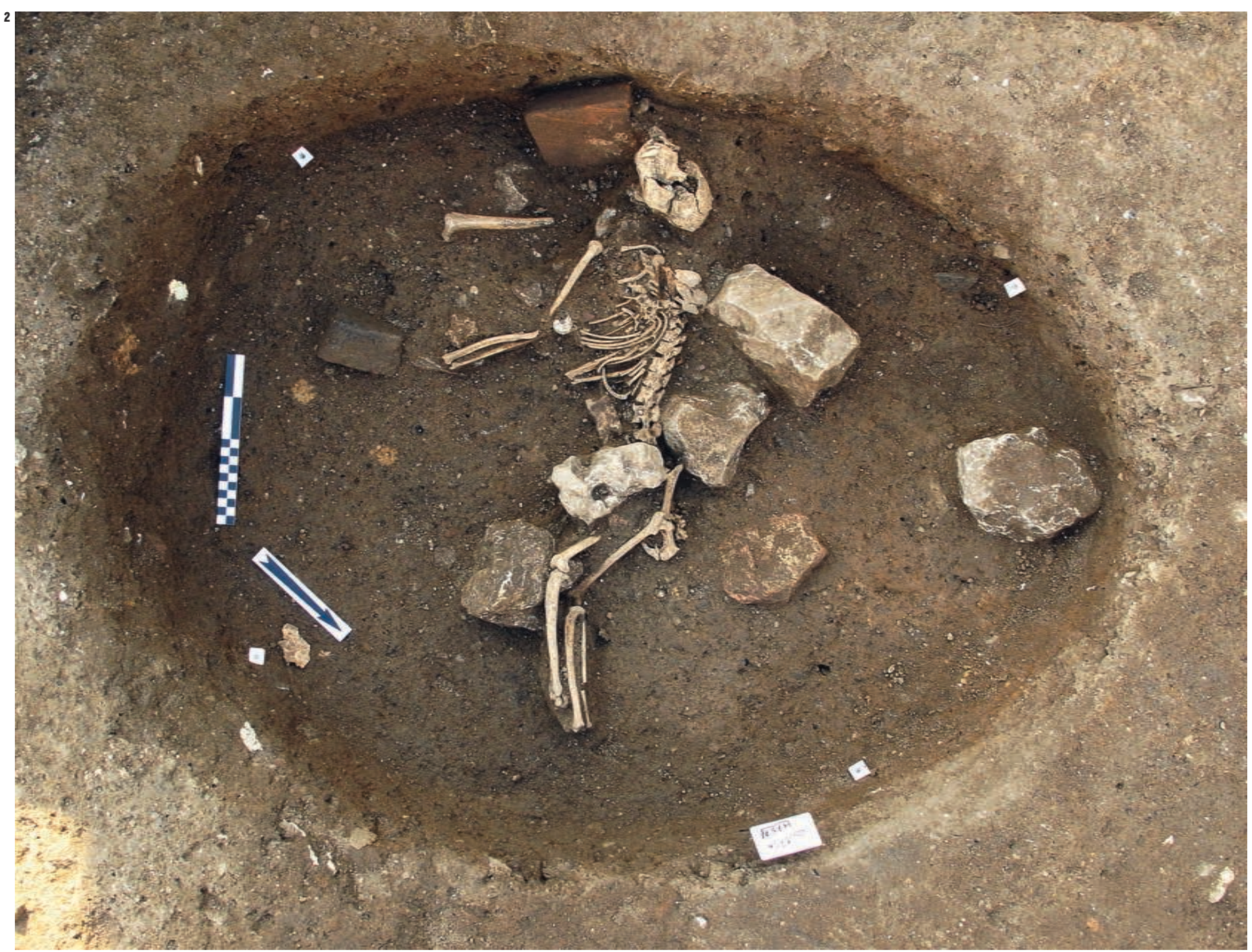




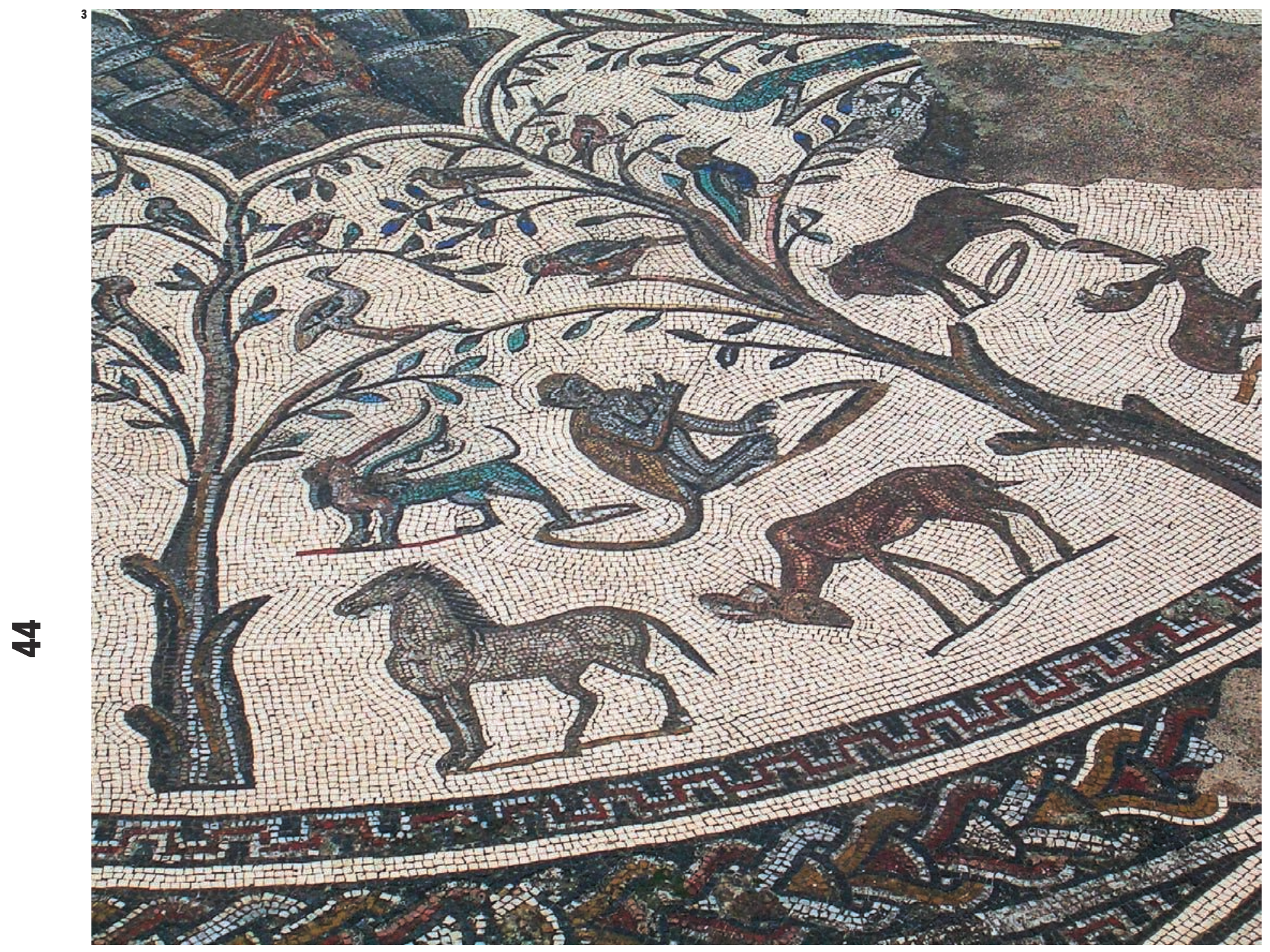

3. Singe en liberté (dans

le panneau central, Orphée

charme les animaux avec

sa lyre). Mosaïque du Tablinium

de la Maison d'Orphée, site

de Volubilis (Maroc). 


\section{Les trois singes de Gaule}

On ne connaissait à ce jour que deux autres squelettes de singes en contexte antique en Gaule. Il s'agit, dans les deux cas, d'un magot, aussi appelé macaque de Gibraltar ou encore macaque berbère (Macaca sylvanus L.). Le premier a été découvert à Narbonne dans la Maison au Grand Triclinium du Clos de la Lombarde, datée de la fin du II $^{\mathrm{e}}$-début du $\mathrm{III}^{\mathrm{e}} \mathrm{s}$. Le cadavre avait été jeté dans le puits de la riche domus de Marcus Claudius Aestivo comme 27 bébés humains et des chiens bassets (Sabrié, 2011). Le second cas provient de la nécropole d'agglomération secondaire de Cutry, en Meurthe-et-Moselle (Poplin, 1986 ; Liéger et al., 1997 , p. $87-88$, fig. p. 135$)^{4}$ : il s'agit d'une femelle très âgée, dont les ossements ont été trouvés en 1975 en bordure d'un dépotoir daté de la première moitié du $\mathrm{II}^{\mathrm{e}} \mathrm{s}$. L'animal semble avoir été enterré dans une fosse, en décubitus dorsal, les jambes légèrement fléchies, le bras gauche le long du corps (le bras droit était manquant, la tombe ayant été recoupée par une autre structure). L'orientation générale de la tombe est sud-ouest/nord-est, avec la tête au sud-ouest. Aucun mobilier n'était associé à la sépulture. Il est intéressant de noter que, même si quelques sépultures à inhumation ont été trouvées sur ce site, l'essentiel des sépultures humaines étaient des incinérations.

\section{Le singe chez les Romains}

Plusieurs espèces de singes, essentiellement de petite taille (pithecium), étaient connues des Romains (McDermott, 1938). Elles provenaient selon Pline, au I ${ }^{\text {er }}$ siècle, et selon Solin, aux III $^{\mathrm{e}}$ et IV ${ }^{\mathrm{e}}$ siècles, d'Éthiopie, d'Égypte et de Libye, et ces auteurs mentionnent leur existence en Inde. Le babouin, avec son aspect mi-chien, mi-singe d'où il tire son nom de cynocephalus (« tête de chien »), semble avoir été traité à part. Alors que les singes les plus couramment représentés appartiennent à la famille des cercopithèques tels que les macaques [ill. 3] ; le seul retrouvé en contexte archéologique est le magot, ou macaque berbère, étant le seul à avoir une queue courte, voire quasi inexistante pour la femelle.

Le singe faisait partie des animaux familiers (de la familia, qui vit ou peut entrer dans la demeure) comme en témoignent la littérature latine (Chevalier, 1995 ; Amat, 2002 ; Lepetz, Yvinec, 2002) et les représentations (King, 2002 ; Greenlaw, 2011); les Romains ont repris en cela une tradition grecque et antérieurement égyptienne et mésopotamienne. Le singe se promenait librement dans la domus, comme en témoigne l'intrigue de la comédie de Plaute, Miles Gloriosus, qui se déroule à Athènes, dans laquelle un adultère est découvert par un esclave qui court après le singe familier sur le toit de la maison du voisin (Amat, 2002, p. 109). La douteuse réputation de l'animal n'empêchait pas les riches propriétaires d'en faire l'acquisition afin de l'exhiber auprès de leurs invités. En effet, l'animal avait un statut ambigu, tour à tour considéré comme une amusante caricature humaine, un animal fourbe, ou une bête méchante. L'animal sera ensuite diabolisé aux débuts de la christianisation, dénoncé comme un être vil et bestial qui imite l'homme, comme le diable tente d'imiter Dieu (Herzfeld, 2012). Chez les auteurs antiques, le singe est souvent utilisé pour ridiculiser quelqu'un que l'on considère comme un pâle imitateur ou un plagiaire. Malgré cela, plusieurs auteurs, comme Martial (Épigrammes, Livre VII, LXXXVII), témoignent de l'engouement que provoquaient les petits singes chez leur maître. On l'habillait souvent en humain [ill. 4] : « La Gaule t'envoie pour vêtement une casaque de Saintonge, ornée d'un capuchon : naguère on en affublait les singes. » (Martial, Épigrammes, Livre XIV, CXXVIII). On le promenait dans les processions, qu'elles soient religieuses comme en témoigne Apulée pour le culte isiaque (Métamorphoses, Livre XI, VIII), ou privées, à l'instar de celle de Publius Verus qu'évoque Cicéron. Comme en Égypte, en Mésopotamie et en Grèce, le thème du singe musicien semble récurrent dans les différentes formes de représentations et dans les textes.

\section{... et dans l'Empire}

Même si aucun auteur antique n'évoque la présence de singes dans les domus de Gaule, les découvertes archéologiques de Narbonne et Poitiers montrent que cette mode n'est pas restée limitée à l'Italie où l'on ne connaît d'ailleurs qu'un seul squelette de singe trouvé à Pompéi (Ciarallo, De Carolis 1999 ; Bailey et al. 1999). En Allemagne, un fragment d'humérus de magot adulte a été découvert dans le vicus de Rainau-Buch. D'autres découvertes en Angleterre en témoignent également. Le crâne et des éléments du squelette d'un jeune mâle magot (Macaca Sylvanus L.) ont été trouvés dans une latrine à Dunstable (Waterman, Lynn, 1997) et sont datés de la fin du $\mathrm{II}^{\mathrm{e}} \mathbf{s}^{\mathbf{6}}$. Pour le Dr Napier, qui en a fait l'identification, il n'y a aucun doute qu'il s'agissait d'un animal de compagnie. Une phalange de magot a également été découverte dans les niveaux tardifs du site anglais antique de Wroxeter (Armour-Chelu, 1997). Cet engouement pour le macaque semble remonter à la fin de l'âge du Fer (Waterman, Lynn, 1997, p. 120-124) si l'on se fie à la découverte d'un crâne et d'une mandibule à Navan Fort, en Irlande du Nord, dont la datation au C14 est établie entre 250 et 100 avant notre ère.

Cet animal de compagnie ne se retrouve toutefois pas uniquement dans les riches demeures. Il a été également la mascotte de certains militaires comme le relate Juvénal (Satires, V), évoquant un singe monté sur une chèvre à qui on apprend à lancer le javelot. On songe là immédiatement à la sépulture découverte en 2001 en Espagne, aux Colomines, à Llivia en Cerdagne. Il s'agit des restes d'un singe africain, un macaque (Macaca sylvanus L.), accompagnés d'un trousseau de diverses pièces de métal dont une boucle de ceinturon de tradition militaire du Bas-Empire, datée des $\mathrm{V}^{\mathrm{e}} \mathrm{ou} \mathrm{VI}^{\mathrm{e}} \mathrm{s}$. carré G11-1. Étude

de Thérèse Poulain.

les interprétations

de 1973 à 1982

au Musée d'Art et

Merci à Vincent

Lamarque, Sébastien

pour les références sur

la tombe de Cutry.

5. «Si Cronius aime un

6. Le mobilier céramique

associé est daté des

années 130-150. 


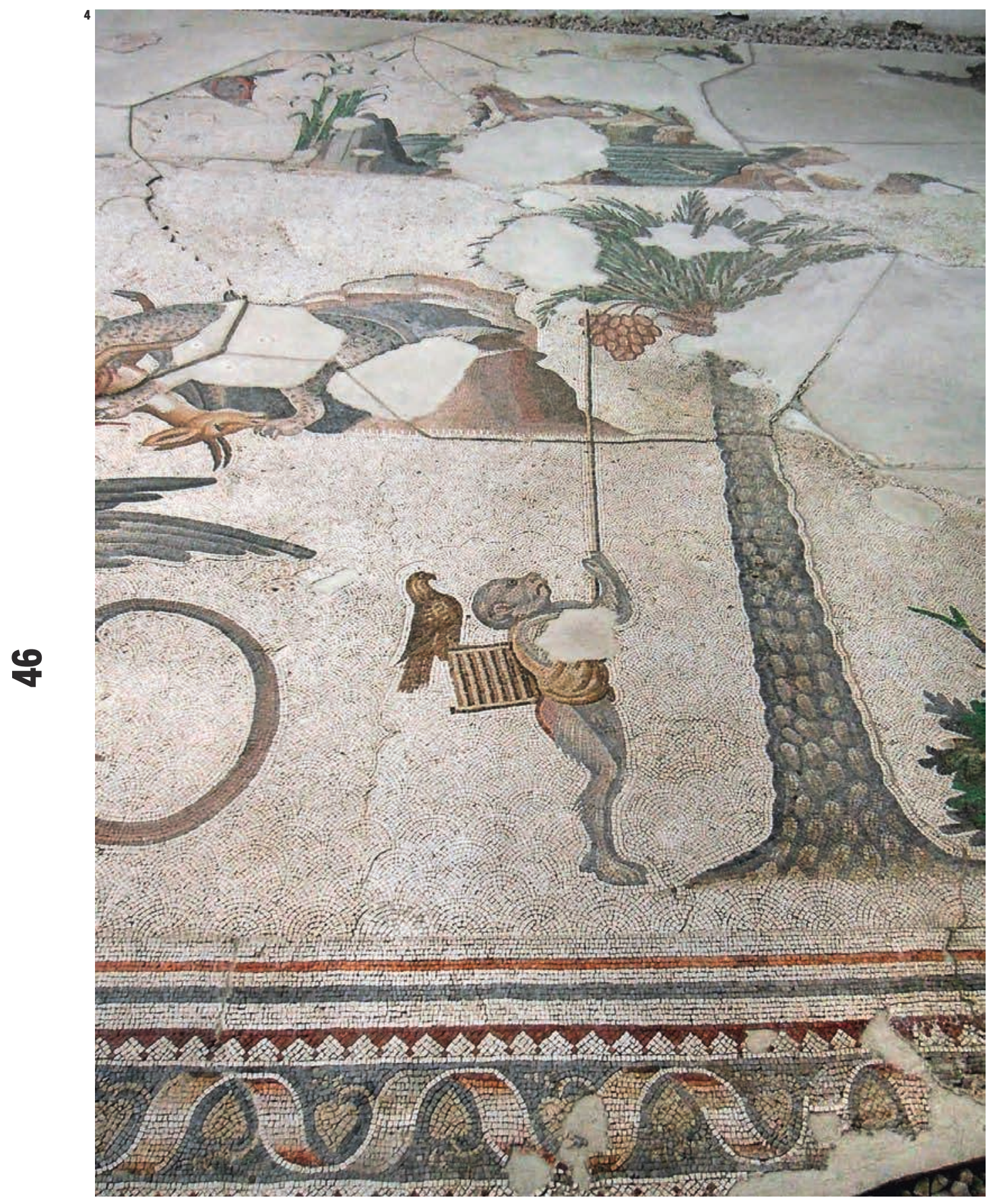

4. Le singe et l'oiseau,

mosaïque du hall nord-est

du grand palais de Byzance

(registre I, secteur C.

panneau (Co), $v^{e}-v^{e} s$

Un singe, très probablement

un magot, vêtu d'un manteau

porte sur son dos une cage

sur laquelle est posé

un oiseau. Il essaye de faire

tomber avec une longue

perche les dattes accrochées

en haut d'un palmier. 
(Guardia et al., 2007). Le site est interprété comme une station de garnison de légionnaires chargés de contrôler un passage à travers les Pyrénées orientales. La fosse quadrangulaire, de 1 sur 0,25 m pour une profondeur minimale de $0,15 \mathrm{~m}$, orientée ouest/est, avait été creusée spécialement pour y enterrer le singe, déposé en décubitus dorsal, les mains croisées sur le pubis, les jambes fléchies sur le côté gauche. Sur le site de Catterick Fort, dans le Yorkshire, construit peu après l'an 70 et occupé jusqu'au III ${ }^{\mathrm{e}}$ s., point stratégique important sur la frontière nord du territoire conquis par les Romains, a été découvert un crâne de magot mâle (Macaca Sylvanus L.) âgé de 3 ans à 3 ans et demi (Napier, 1981; Masseti, Brunner, 2009; Wilson, 2002). Il n'est pas certain toutefois que le singe était dans un contexte militaire. Abel Liéger avait émis l'hypothèse que le magot de Cutry avait été rapporté par un légionnaire. Aucun élément cependant ne permet de confirmer cette hypothèse qui n'est d'ailleurs pas reprise dans la publication de la nécropole (Liéger, 1997).

L'enterrement du singe des Caillons dans l'enclos funéraire le fait passer du statut de simple animal exotique à celui de membre à part entière de la famille. En effet, pour le droit romain, seul les plus proches parents, les enfants émancipés et les héritiers (qui peuvent ne pas être de la famille, mais qui ont été désignés comme tels) peuvent y prendre place (Daniel-Lacombe, 1886). Les affranchis sont également admis. Tout membre de la famille (au sens latin du terme) a donc le droit d'être enterré dans cet espace. Même si les descriptions naturalistes comme celles de Pline mettent surtout en avant le caractère utile de l'animal, les portraits peints ou sculptés, certains monuments funéraires qui leurs sont réservés ou les éloges littéraires montrent la dimension psychologique et affective de la relation entre le maître ou l'utilisateur et son animal (Bodson, 1997). Ainsi, les inhumations de chiens, seuls ou associés à un humain, ne sont pas rares en Gaule, comme celles, un peu moins courantes, de chevaux (Lepetz, 1996). Ces animaux ont bel et bien une place particulière qui les met à part des autres espèces. Ce statut spécial se retrouve dans des contextes sacrificiels (Méniel, Jouin, 2000 ; Lepetz, 2000 ; Reynaud Savioz, Pignolet, 2006). Les singes n'étaient probablement pas les animaux les plus courants en Gaule et dans les autres provinces de l'Empire, mais il est fort probable que les rares découvertes de magots et autres primates en contexte archéologique réalisées à ce jour ne reflètent pas la réalité du monde antique. La généralisation des études archéozoologiques permettra probablement de compléter un peu le tableau, mais la connaissance de cette réalité augmentera surtout la vigilance des archéologues.

\section{Références bibliographiques}

APUlÉE, SERS O. (TRAD.), 2007 (réédition de 1945), Les Métamorphoses ou l'Âne d'or, Paris, Belles Lettres.

Juvénal, Sers O. (TRAD.), 2002 (réédition de 1921), Satires, Paris, Belles Lettres.

Martial, IzAaC H.J. (TRAD.), 1930 et 1934, Épigrammes, t. I et t. II, $2^{\mathrm{e}}$ partie, Paris, Belles Lettres.

Pline, Ernout A. (TRAD.), 1952 et 1947, Histoire Naturelle, Livre VIII et XI, Paris, Belles Lettres.

Solin, Mommsen TH. (ÉD.), 1958 (réédition de 1895), Collectanea rerum memorabilium, iterum recensivit, Berolini.

Амат J., 2002, Les animaux familiers dans la Rome antique, Paris, Belles Lettres, 284 p.

Armour-Chelu M., 1997, «Appendix 8: faunal remains», in BARKer P. et al., The baths basilica Wroxeter: excavations 1966-9o, Londres, English Heritage, Archaeological Report, 8, p. 350-364.

Bailey J. F., Henneberg M., Colson I. B., Ciarallo A., Hedges R.E.M., SyKes B., 1999, Monkey Business in Pompeii-Unique Find of a Juvenile Barbary Macaque Skeleton in Pompeii Identified Using Osteology and Ancient DNA Techniques, The Journal - Molecular Biology and Evolution - Oxford Journals, 16(10), p.1410-1414.

Bodson L., 1997, «Le témoignage de Pline l'Ancien sur la conception romaine de l'animal », in CASsin B et al., L'animal dans l'Antiquité, «Bibliothèque d'Histoire de la Philosophie», nouvelle série, Paris, Librairie Philosophique J. Vrin, p. 325-354.

BoDSON, L. (ÉD.), 200o, Ces animaux que l'homme choisit d'inhumer. Contribution à létude de la place et du rôle de l'animal dans les rites funéraires, Université de Liège, 20 mars 1999, Colloques d'histoire des connaissances zoologiques, 11, Liège, Université de Liège.
Chevalier R. ÉD, 1995, Homme et animal dans l'antiquité romaine, Actes du colloque de Nantes organisé par le Centre de Recherches André Piganiol, Ecoles vétérinaire de Nantes, 30 mai - 1er juin 1991, Tours, Caesarodunum, H.S., $470 \mathrm{p}$

Ciarallo A., De Carolis E., 1999, Homo faber. Natura scienza e tecnica nell'antica Pompei, Catalogue de l'exposition, Napoli, Museo archeologico Nazionale. 27 marzo - 18 luglio 1999, Milan, Electa, 346 p.

Daniel-Lacombe H., 1886, Le Droit funéraire à Rome, Paris, Alphonse Picard éditeur, 221 p.

GREENLAW C., 2011, The representation of monkeys in the art and thought of Mediterranean cultures: a new perspective on ancient primates, Oxford, Archaeopress, British Archaeological Reports.

GUARDIA J. et al., 2007, « Enterrament d'epoca tardoromana d'un macaco Amb "Aixovar" al jaciment de Les Colomines (Llivia, La Cerdanya)», Empuries, 55, p. 199-227.

HerZFeld C., 2012, Petite histoire des grands singes, Paris, Seuil, «Science ouverte», 210 p.

KING A., 2002, «Mammals. Evidence from wall paintings, sculpture, mosaics, faunal remains, and ancient literary sources », in FEEMSTER JASHEMSKI W., MeYer F.G. (ÉD.), The natural history of Pompeii, Cambridge, Cambridge University Press, p. 401-45o.

LEPETZ S., 1996, «Les animaux dans les pratiques funéraires », Revue archéologique de Picardie, vol. 12, $\mathrm{n}^{\circ} 12$, p. 148-153.

LEPETZ S., 200o, « Sacrifices et inhumations de chevaux et de chiens en France du Nord au III ${ }^{\mathrm{e}}$ siècle après J.-C. », in BODSON L. (ÉD.), op. cit., p. 93-125.

LEPETZ S., YVINEC J.-H., 2002, «Présence d'espèces animales d'origines méditerranéennes en France du Nord aux périodes romaine et médiévale : actions anthropiques et mouvements naturels », in GARDEISEN A. (ÉD.), Mouvements ou déplacements de populations animales en Méditerranée au cours de l'Holocène, Séminaire de recherche "Archéologie de l'Animal », Lattes-Montpellier, 29 Septembre 20oo, BAR International, séries 1017, p. 33-42.

LIÉGER A. (DIR.) et al., 1997, La nécropole gallo-romaine de Cutry (Meurthe-et-Moselle), Études Lorraines d'Archéologie Nationale, 3, Presses Universitaires de Nancy, $237 \mathrm{p}$.

MASSETI M., BrunNer E., 2009, «The primates of the western Palaearctic: a biogeographical, historical, and archaeozoological review », JAS, 87, p. 33-91.

MÉNIEL P., JouIN M., 200o, «Les inhumations d'animaux de Vertault (Côte-d'Or, début de notre ère) " in Bodson L. (ÉD.), op. cit., p. 65-91.

NAPIER P.H., 1981, Catalogue of Primates in the British Museum (Natural History) and elsewhere in the British isles. Part 2. Family Cercopithecidae, subfamily Cercopithecinae, Londres, British Museum.

McDermotT W. C., 1938, The Ape in Antiquity, Baltimore, John Hopkins Press.

Poplin F., 1986, «Introduction aux animaux et aux végétaux du séjour des morts ", in DUdAY H., MASSET C. (DIR.), Actes du colloque Anthropologie physique et Archéologie, méthodes d'étude des sépultures, Toulouse, 4-6 novembre 1982, Paris, éditions du CNRS, p. 281-287.

Reynaud Savioz N., Pignolet M., 2006, «Un cas d'inhumation de chiots gallo-romains (Martigny VS, Forum Claudii Vallensium, la Délèze, insula 5)», Bull. Murithienne, 124, p. 31-38

SAbriÉ M., SAbriÉ R., 2011, La Maison au Grand Triclinium du Clos de la Lombar de à Narbonne, Archéologie et Histoire Romaine, 19, éd. Monique Mergoil, 396 p., 310 fig., XXXII pl.

WATERMAN D.M., LYNN C.J., 1997, Excavations at Navan Fort 1961-71, County Armagh, Northern Ireland Archaeological Monographs, 3, Belfast, Stationery Office. 